\title{
Influence of synergism with Bacillus thuringiensis and Beauveria bassiana on diamondback moth larvae
}

\author{
Xue Chun-Mei 1,a, Sun Yan-Bin ${ }^{2, b}$, Zhang Yue-Hua ${ }^{2, c *}$ \\ ${ }^{1}$ College of Life Science, Jiamusi University Jiamusi, 154007, PR China; \\ ${ }^{2}$ College of Science, Jiamusi University Jiamusi, 154007, PR China \\ ajmsxcm@126.com, b63145964@qq.com, ${ }^{* *}$ Corresponding Author:751520177@.com
}

Key word: Bacillus thuringiensis; Beauveria bassiana ;Compound; Plutella xylostella

Abstract: [Objective] Researching the effects of different concentrations of $B$. thuringiensis and $B$. bassiana mixed liquor on the mortality of Plutella xylostella larvae in Jiamusi. [Methods] Employing PBS buffer diluted the B. thuringiensis and B. bassiana into different concentrations of suspension, the mixture of $B$. thuringiensis and $B$. bassiana was mixed into seven different concentration gradients by orthogonal analysist, and determination the mortality of P. xylostella larvae at different concentrations, and then screened more suitable larval $\mathrm{LD}_{50}$ proportion and concentration. [Results] According to the observation and analysis, when the ratio of $B$. thuringiensis and B. bassiana was 3: 1 in 96h, the P. xylostella larvae mortality rate reached $72 \%$, among them, the median lethal rate of larvae in $10^{-1} \sim 10^{-6} \mathrm{~g} / \mathrm{mL}$ was higher. [Conclusion] The lethal effect of each mixture on the diamondback moth larvae was different. When the mixture ratio of $B$. thuringiensis and B. bassiana was 3: 1 and the concentration was $10^{-1} \sim 10^{-6} \mathrm{~g} / \mathrm{mL}$, the lethal effect on insect larvae was obvious.

\section{Introduction}

Lepidoptera insects Plutella xylostella larvae mainly endanger cabbage, broccoli and other vegetables ${ }^{[1]}$, resulting in decreased vegetable production, farmers reduce income. In the process of pesticide removal in the field, chemical control and biological control methods are commonly used. However, unreasonable application of chemical pesticides has resulted in serious environmental pollution $^{[2]}$, which affects the quality and output of vegetables to varying degrees ${ }^{[3]}$. $B$. thuringiensis is an effective microbial insecticide in biological control. It can produce insecticidal crystal protein, $\alpha$-exotoxin, $\beta$-exotoxin and other substances in the process of growth and metabolism $^{[4]}$. Insecticidal rate, no pollution to humans and animals and the environment, etc ${ }^{[5]}$. But there are easy to produce resistance, persistent and poor stability and other drawbacks. However, Beauveria bassiana has the characteristics of wide distribution, strong infectivity and epidemic spread in nature. However, due to the unstable quality and application effect of Beauveria bassiana powder and its insecticidal speed Slower, affecting the enthusiasm of forest farmers to use. $B$. thuringiensis and B. bassiana alone have some limitations. In this paper, the B. thuringiensis and $B$. bassiana mixed with seven different concentrations of gradient mixture on the diamondback moth larvae mortality in order to use biological control measures to control field pests, which agricultural pollution prevention and control of Northeast China and the promotion of biological pesticides are of great significance. 


\section{Materials and methods}

Test materials

Xian lou Bacillus thuringiensis powder (Bt) and Tian li Beauveria bassiana powder (Bb). Plutella xylostella larvae collected from Jiamusi City, Heilongjiang Province, Jiamusi University Jianan Experimental Farm vegetable cultivation experiment base.

Medium production

Beef extract peptone medium: beef extract $5.0 \mathrm{~g} / \mathrm{L}$, peptone $10.0 \mathrm{~g} / \mathrm{L}, \mathrm{NaCl} 5.0 \mathrm{~g} / \mathrm{L}$, agar $20.0 \mathrm{~g} / \mathrm{L}$, distilled water volume to $1.0 \mathrm{~L}, \mathrm{pH} 7.2-7.4$. PDA medium: potato $200.0 \mathrm{~g} / \mathrm{L}$, glucose $20.0 \mathrm{~g} / \mathrm{L}$, agar $20.0 \mathrm{~g} / \mathrm{L}$, distilled water volume to $1.0 \mathrm{~L}, \mathrm{pH}$ natural.

PBS buffer preparation

Weigh $8.0 \mathrm{gNaCl}, 0.2 \mathrm{gKCl}, 3.63 \mathrm{gNa} 2 \mathrm{HPO} 4 \cdot 12 \mathrm{H} 2 \mathrm{O}, 0.24 \mathrm{gKH} 2 \mathrm{PO} 4$ dissolved in $900 \mathrm{~mL}$ distilled water, with hydrochloric acid to adjust the $\mathrm{pH}$ to 7.4 , water $1.0 \mathrm{~L}$.

Sterile buffer preparation

The PBS buffer and distilled water, $1.0 \mathrm{~mL}$ pipette tip and the prepared medium together into an autoclave at a pressure of $121^{\circ} \mathrm{C}, 0.1 \mathrm{MPa}$ sterilization $20 \mathrm{~min}$. Under sterile conditions $B$. thuringiensis and B. bassiana were weighed $1.000 \mathrm{~g}$ with analytical balance, into $10.0 \mathrm{~mL}$ PBS buffer, the concentration of $10^{-1} \mathrm{~g} / \mathrm{mL}$, followed by dilution to $10^{-10} \mathrm{~g} / \mathrm{mL}$ and Set up a control experiment, concentration gradient dilution with sterile distilled water, each experiment was repeated 2 times.

Strains coated

The suspension of $B$. thuringiensis concentration of each gradient was applied to $15.0 \mathrm{~mL}$ beef extract peptone medium. B. bassiana suspension of each concentration gradient was applied to $15.0 \mathrm{~mL}$ PDA medium, and control experiments were designed with distilled water, experimental group and control group repeated 5 times. The coated uniform culture dish into the incubator, $29^{\circ} \mathrm{C}$ for 2 days.

Mixed liquid preparation

B. thuringiensis and B. bassiana were made into a single dose and mixed agent, the mixture Bt: Bb codenamed $\mathrm{A}_{1}, \mathrm{~A}_{2}, \mathrm{~A}_{3}, \mathrm{~A}_{4}, \mathrm{~A}_{5}$, the ratio was 1:1,1:2, 2:1, 1: 3 and 3:1.

Indoor biological determination

The Plutella xylostella larvae were immersed in different concentrations of the mixture for $10 \mathrm{~s}$, air-dried, placed in a diameter of $9 \mathrm{~cm}$ Petri dishes, each with a mixture of 10 treated with distilled water as a control. The collected fresh young cabbage leaves were washed dry weighed $2.0 \mathrm{~g}$ were dipped into different mixes 10s, removed into the Petri dishes. Placed at $25^{\circ} \mathrm{C}$ feeding, daily replacement of fresh young cabbage leaves, respectively, at 24h, 48h, 72h and 96h statistics larval mortality. All data are processed using SPSS16.60 software. 


\section{Result analysis}

Buffer effect comparison

B. thuringiensis and B. bassiana were diluted with PBS buffer solution and distilled water for $24 \mathrm{~h}$, respectively. The numbers of Bt and Bb colonies diluted with PBS buffer were $298 \mathrm{cfu} / \mathrm{mL}$ and $276 \mathrm{cfu} / \mathrm{mL}$. The number of colonies diluted with distilled water was $224 \mathrm{cfu} / \mathrm{mL}$ and $210 \mathrm{cfu} / \mathrm{mL}$, respectively, and the number of colonies diluted with PBS was significantly higher than that of distilled water, indicating that PBS buffer was more diluted than distilled water Liquid better protect the integrity of microorganisms (see Table 1).

Table 1. B. thuringiensis and B. bassiana were diluted in water and PBS, respectively

\begin{tabular}{|c|c|c|c|c|c|c|c|c|c|c|c|}
\hline \multirow{2}{*}{$\begin{array}{c}\text { The fungus } \\
\text { powder }\end{array}$} & \multirow{2}{*}{ Buffer } & \multicolumn{9}{|c|}{ concentration $[\mathrm{g} / \mathrm{mL}]$} & \multirow[b]{2}{*}{$10^{-10}$} \\
\hline & & $10^{-1}$ & $10^{-2}$ & $10^{-3}$ & $10^{-4}$ & $10^{-5}$ & $10^{-6}$ & $10^{-7}$ & $10^{-8}$ & $10^{-9}$ & \\
\hline \multirow[t]{2}{*}{$\mathrm{Bt}$} & $\begin{array}{c}\text { Distilled } \\
\text { water }\end{array}$ & 224 & 178 & 156 & 104 & 65 & 48 & 31 & 18 & 9 & 3 \\
\hline & PBS & 298 & 250 & 202 & 152 & 129 & 86 & 52 & 27 & 12 & 6 \\
\hline \multirow[t]{2}{*}{$\mathrm{Bb}$} & $\begin{array}{c}\text { Distilled } \\
\text { water }\end{array}$ & 210 & 165 & 104 & 85 & 65 & 34 & 22 & 9 & 3 & 1 \\
\hline & PBS & 276 & 233 & 165 & 103 & 67 & 44 & 32 & 11 & 5 & 2 \\
\hline
\end{tabular}

\section{Determination of different mixture properties}

The result of applying $4 \mathrm{~d}$ mixture showed that the mixture of B. thuringiensis and B. bassiana mixed with 7 kinds of mixture with different proportions was higher than that of single dose of $\mathrm{Bt}$ and single dose of $\mathrm{Bb}$. The larvae did not die within 24 hours. The mortality rate of single dose of $\mathrm{Bt}$ was higher than that of single dose of $\mathrm{Bb}$ within 48 hours. The mortality rate was $16 \%$ under the $\mathrm{Bt}: \mathrm{Bb}=1: 3$ ratio and the mortality rate under $\mathrm{Bt}: \mathrm{Bb}=2: 1$ ratio The mortality rate of larvae reached $15 \%$ at $22 \% \mathrm{Bt}: \mathrm{Bb}=3: 1$, and the larval mortality increased within 72 hours. At this time, the dead larvae lost their luster compared with the control larvae, There are dark brown spots of varying sizes. No mortality was observed in the $96 \mathrm{~h}$ control group. The mortality rate was as high as $72 \%$ for the $\mathrm{Bt}: \mathrm{Bb}=3: 1$ ratio, exceeding $15 \%$ for the $\mathrm{Bt}: \mathrm{Bb}=1: 3$ ratio (Figure 1 ).

\section{$2.3 \mathrm{Bt}: \mathrm{Bb}=3: 1$ the ratio of the concentration of each comparison}

Experimental data show that with the extension of time, the mortality of diamondback moth larvae gradually increased. When the ratio of $B$. thuringiensis and B. bassiana was 3: 1, the larvae did not die within 24 hours. The larvae gradually died within $10^{-1} \mathrm{~g} / \mathrm{m} \sim 10^{-8} \mathrm{~g} / \mathrm{mL}$ within $48 \mathrm{~h}$, and larvae died within $72 \mathrm{~h}$ Gradually increased within $96 \mathrm{~h} 10^{-1} \mathrm{~g} / \mathrm{mL} \sim 10^{-6} \mathrm{~g} / \mathrm{mL}$ concentration has reached $60 \%$ to $80 \%$, to achieve the experimental determination of the standard ( Figure 2 ).

\section{Conclusion analysis}

According to the indoor toxicity test of B. thuringiensis and B. bassiana on the common pests in North conservatory, the results showed that the mortality of Plutella xylostella larvae was higher within $96 \mathrm{~h}$ under the $\mathrm{A}_{5}$ ratio, The mortality of larvae was between $60 \%$ and $70 \%$ at the concentration of $10^{-5} \mathrm{~g} / \mathrm{mL} \sim 10^{-6} \mathrm{~g} / \mathrm{mL}$. Comparing the results of experiment and production cost, the two concentrations accord with the standard of this experiment and could be applied in the process of removing pesticide in field. 

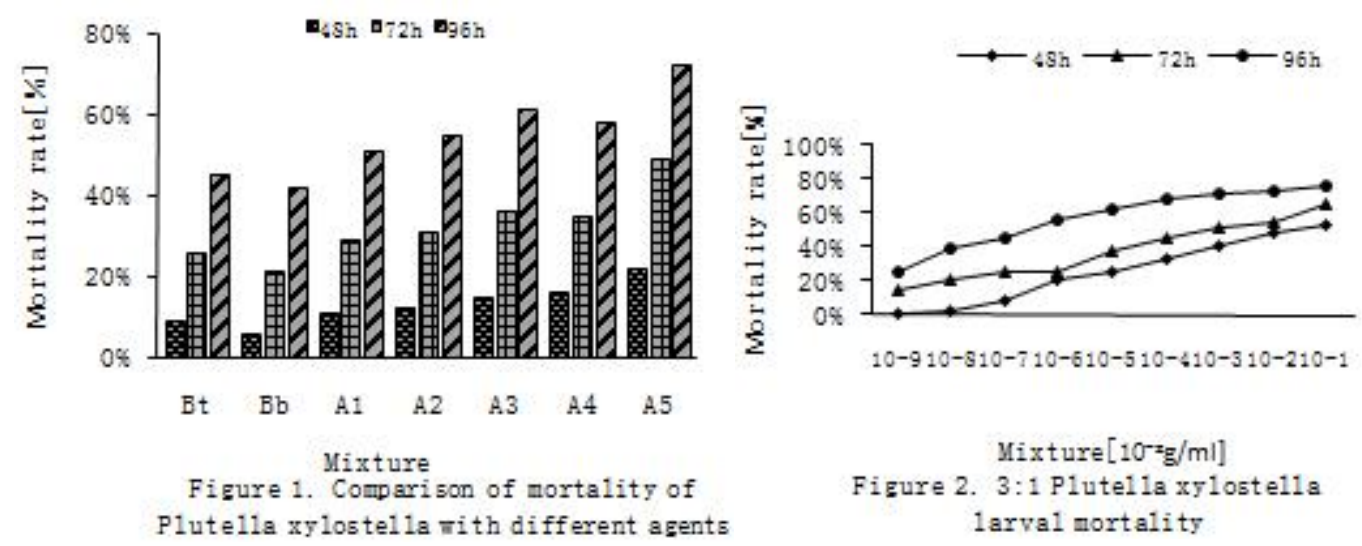

In this experiment, the virulence of the northern pests were determined, the application of $B$. thuringiensis and B. bassiana in the field planting and fruit tree planting can effectively prevent and reduce pest insects such as diamondback moth caused by the phenomenon of production, but the growth of field pests in the environment There is a certain gap between the indoor environment and the natural environment for the growth of insects, and the effect of $B$. thuringiensis and $B$. bassiana is easily affected by the environmental conditions such as ultraviolet radiation, light and air temperature, and the related research is still needed in practical application .

\section{Acknowledgements}

Heilongjiang Provincial Nature Fund Project (C2017064); Jiamusi University Science research Project: JMSUJCMS2016-015.

\section{References:}

[1] Furlong, Michael J., Denis J. Wright, and Lloyd M. Dosdall. "Diamondback moth ecology and management: problems, progress, and prospects." Annual Review of Entomology 58 (2013): 517-541.

[2] Shimoda, Masami, and Ken-ichiro Honda. "Insect reactions to light and its applications to pest management." Applied Entomology and Zoology 48.4 (2013): 413-421.

[3] Lu, Yonglong, et al. "Impacts of soil and water pollution on food safety and health risks in China." Environment international 77 (2015): 5-15.

[4] Li, Wen-Wei, and Han-Qing Yu. "From wastewater to bioenergy and biochemicals via two-stage bioconversion processes: a future paradigm." Biotechnology Advances 29.6 (2011): 972-982.

[5] Chen, Hao, YongJun Lin, and QiFa Zhang. "Review and prospect of transgenic rice research." Chinese Science Bulletin 54.22 (2009): 4049. 